\title{
Identification and Composition of Emerging Quaternary Ammonium Compounds in Municipal Sewage Sludge in China
}

\author{
Ting Ruan, Shanjun Song, Thanh Wang, Runzeng Liu, Yongfeng Lin, and Guibin Jiang*
}

State Key Laboratory of Environmental Chemistry and Ecotoxicology, Research Center for Eco-Environmental Sciences, Chinese Academy of Sciences, Beijing 100085, People's Republic of China

\section{Supporting Information}

\begin{abstract}
Quaternary ammonium compounds (QACs) have raised considerable attention due to their wide commercial applications and recent discovery of unknown persistent analogues in aqueous environment. In this work, the occurrence and distribution of alkyltrimethylammonium (ATMAC), benzylakyldimethylethylammonium (BAC) and dialkyldimethylammonium (DADMAC) homologues were investigated in fifty-two municipal sewage sludge samples. ATMAC C10-18, BAC C8-18 and paired DADMAC C8:8-C18:18 as well as emerging homologues such as ATMAC-20, 22 and mixed DADMAC-16:18 and 14:16 were present. Furthermore, paired DADMAC-20:20 and mixed DADMAC-14:18, 18:20 were identified for the first time by nontarget qualitative strategies. A triple quadruple mass spectrometer quantification method was also initially verified with the aid of laboratory synthesized standards for the analysis of the mixed DADMACs with no certificated commercial standards currently available. The total concentrations of ATMACs, BACs and DADMACs were in the range of $0.38-293,0.09-191$ and $0.64-344 \mu \mathrm{g} / \mathrm{g}$ dry weight, respectively, and particularly, mixed DADMACs constituted $39 \pm 7 \%$ of total DADMAC concentrations. The concentrations and profiles of individual homologues further suggested different QAC applications and fate in China. Significant correlations were also found among the concentrations of various QAC homologues as well as wastewater treatment plant (WWTP) characteristics (total organic carbon contents and daily treatment volumes).

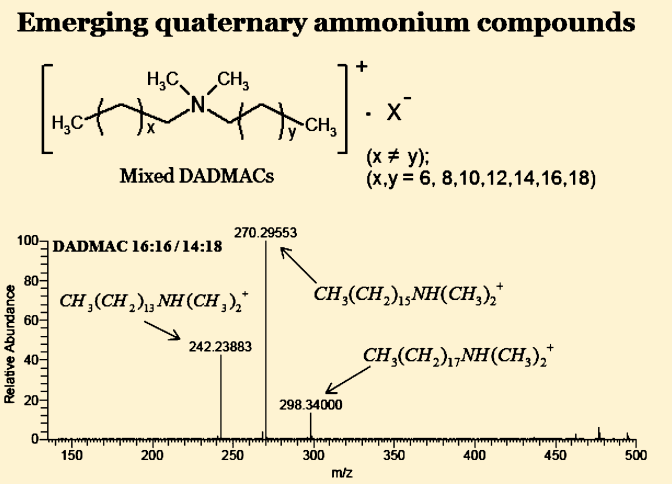

Emerging quaternary ammonium compounds
\end{abstract}

\section{INTRODUCTION}

Rapid development of analytical methods and increasing public health concerns have improved the assessment and regulatory strategies for screening chemicals with persistent, bioaccumulative, and toxic potentials, especially from the high production volume chemical (HPVC) list. $^{1-5}$ Surfactants are an economically important class of HPVCs, with wide consumer usages and industrial applications. The transport, fate, and possible adverse effects of some important neutral and anion surfactants, such as nonylphenolethoxylates and relevant metabolites, have been extensively investigated in various environmental compartments. ${ }^{6,7}$ However, less attention has been raised on cationic surfactants, although some of these could be environmentally persistent as they result from the amphiphilic and electrostatic interactions with negatively charged surfaces and are potentially toxic to aquatic organisms. ${ }^{8-12}$

Here, we focused our research on quaternary ammonium compounds (QACs), a class of cationic surfactants that are commonly used as detergents, phase transfer catalysts, fabric softeners, disinfectants as well as preservatives and antielectrostatics in personal care products. ${ }^{8,13}$ QACs mainly consist of the alkyltrimethylammonium (ATMACs), benzylalkyldimethylethylammonium (BACs), and dialkyldimethyl-ammonium compounds (DADMACs) as shown in Figure 1. The analysis and ecological risk assessment of QACs in the environment began

pubs.acs.org/est 


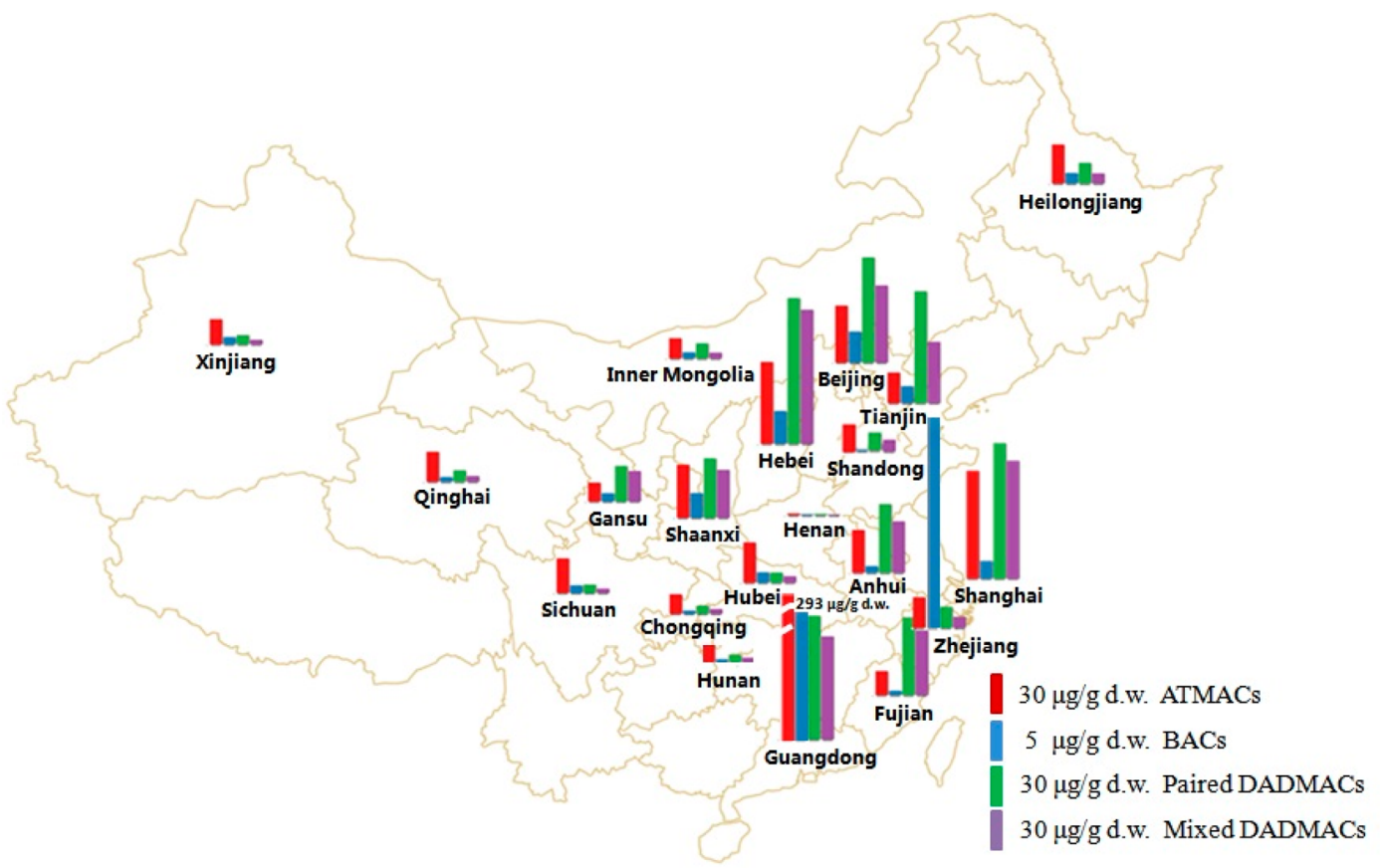

Figure 2. Sampling locations and spatial distributions of quaternary ammonium compound concentrations in municipal sewage sludge samples in China (mean concentration values of different QAC analogues were present for WWTP samples in the same provinces/municipalities).

and other local point sources, such as hospitals, ${ }^{22}$ laundry wastewater, $^{9}$ and roof runoff, ${ }^{23}$ which resulted in a voluntary phase-out of DADMACs in European countries and subsequent concentration reduction in wastewater treatment plants (WWTPs). ${ }^{17}$ Nevertheless, QAC analogues are still frequently found in freshwater sediment by nontarget screening protocols for priority pollutants, ${ }^{24,25}$ which further suggests the potential persistency of QACs in the environment. Other emerging hydrophobic analogues, such as ATMAC-22 as well as mixed DADMAC-14:16 and 16:18, have also recently been identified in urban estuarine sediment, ${ }^{8,17,26}$ which accounted for a substantial proportion of ATMACs and DADMACs and suggested that the total QAC residue levels might have been underestimated. However, there is still very limited research on the presence, distribution, and fate of these emerging QAC analogues in different environment compartments.

Wastewater treatment plants can be a major sink of many organic contaminants. Sewage sludge is commonly considered as a unique medium for tracking various emerging aqueous contaminants, which could re-enter the ambient environment through biosolids application. ${ }^{27-30}$ Due to cationic surfactant properties, QAC residues could also affect the solubility and mobility of other coexisting organic pollutants in the sludgeamended compartments. ${ }^{27,31,32}$ In this work, quantification strategies for the trace analysis of ATMAC, BAC, and DADMACs (including mixed homologues) were developed, and municipal sewage sludge samples were also analyzed in order to provide valuable information on the existence and distribution of various QAC analogues in different Chinese cities. To our knowledge, this is the first work to report of the levels of several emerging QACs in sewage sludge in China.

\section{MATERIAL AND METHODS}

Materials. Chemical name, acronym, structure and other information are shown in Supporting Information, SI, Table S1. Benzylalkyldimethylethylammonium chloride (BAC-8, 10, 12,
14, 16, 18) were purchased from Sigma-Aldrich (St. Louis, MO). Alkyltrimethylammonium bromide (ATMAC-10, 12, 14, 16, 18) and paired dialkyldimethylammonium bromide (DADMAC-8:8, 10:10, 12:12, 14:14, 16:16, 18:18) were from TCI (Tokyo, Japan). Mixed DADMAC-14:18 (purity 97\%), 16:18 (96\%) and 18:20 (97\%) were laboratorysynthesized with detailed synthesis procedures given in the SI. Isotope-labeled internal standards (IS) D7-BAC-14, and D7BAC-18 were obtained from Toronto Research Chemicals (North York, ON, Canada). Sodium octadecylbenzenedulfonate was from Sinopharm Chemical Reagent Inc. (Beijing, China). The purities of all chemicals were $96 \%$ or higher. AG1$\mathrm{X} 2$ anion exchange resin was purchased from Bio-Rad (chloride form, 50-100 mesh, Hercules, CA). HPLC-grade methanol, chloroform, and concentrated hydrochloric acid (37\%) were separately obtained from J.T. Baker (Phillipsburg, NJ) and Merck (Darmstadt, Germany). Ultrapure water $(18.2 \mathrm{M} \Omega \cdot \mathrm{cm})$ was generated by a Milli-Q system (Millipore, Billerica, MA).

Sampling and Extraction Procedures. The sampling map and sites are shown in Figure 2, and the details have been published elsewhere. ${ }^{29}$ Briefly, one grab sewage sludge samples were collected during October 2010 to May 2011 from each of 52 municipal wastewater treatment plants in 20 provinces and municipalities in China. Approximately $500 \mathrm{~g}$ wet weight of the freshly digested sludge sample obtained from WWTP dehydration process was packed in aluminum foil, sealed in Ziplock bags and express mailed to our laboratory. All samples were then freeze-dried, homogenized, and stored at $-20{ }^{\circ} \mathrm{C}$ until sample pretreatment. Relevant information of each WWTPs in regards to type of treatment and treatment processing volume is summarized in SI Table S2.

The detailed sample pretreatment procedures were similar to those in previous reported literature, ${ }^{17,20}$ with minor modifications. Before use, all glassware was soaked in noncationic detergent (Decon 90, Decon Laboratories Limited, East Sussex, UK) overnight to avoid analyte loss by adsorption 
Table 1. Descriptive Statistics of the Measured QAC Concentrations ( $\mu \mathrm{g} / \mathrm{g}$ d.w. Sludge) in the Investigated Sludge Samples

\begin{tabular}{|c|c|c|c|c|c|c|c|c|}
\hline \multirow[b]{2}{*}{ variable } & & \multirow[b]{2}{*}{$\mathrm{GM}^{a}$} & \multirow[b]{2}{*}{ median } & \multirow[b]{2}{*}{ 95th percentage } & \multirow[b]{2}{*}{ range } & \multirow[b]{2}{*}{ quantification rate $^{b}(\%)$} & \multicolumn{2}{|c|}{ averaged proportion (\%) } \\
\hline & & & & & & & in each analogue & in $\Sigma$ QACs \\
\hline \multicolumn{9}{|l|}{ ATMACs } \\
\hline & C10-C14 & 0.24 & 0.17 & 2.03 & $0.04-3.47$ & 100 & 2.7 & 0.7 \\
\hline & $\mathrm{C} 16-\mathrm{C} 18$ & 8.11 & 9.68 & 103 & $0.16-180$ & 100 & 52.7 & 20.6 \\
\hline & $\mathrm{C} 20-\mathrm{C} 22$ & 6.43 & 8.59 & 47.9 & $0.17-111$ & 100 & 44.6 & 18.2 \\
\hline & $\Sigma$ ATMACs & 16.0 & 18.9 & 137 & $0.38-294$ & 100 & 100 & 39.5 \\
\hline \multicolumn{9}{|l|}{ BACs } \\
\hline & C8-C10 & 0.01 & 0.01 & 0.03 & $<\mathrm{LOQ}-0.81$ & 3.8 & 0.6 & 0.0 \\
\hline & $\mathrm{C} 12-\mathrm{C} 16$ & 1.29 & 0.96 & 16.5 & $0.87-188$ & 100 & 87.2 & 5.3 \\
\hline & C18 & 0.13 & 0.13 & 2.18 & $<\mathrm{LOQ}-3.23$ & 96.2 & 12.2 & 0.5 \\
\hline & $\Sigma$ BACs & 1.49 & 1.09 & 17.6 & $0.94-191$ & 100 & 100 & 5.9 \\
\hline \multicolumn{9}{|l|}{ DADMACs } \\
\hline & $\mathrm{C} 8: \mathrm{C} 8-\mathrm{C} 10: 10^{c}$ & 0.17 & 0.18 & 1.93 & $0.02-3.52$ & 100 & 1.5 & 0.6 \\
\hline & $\mathrm{C} 12: \mathrm{C} 12-\mathrm{C} 18: 18^{c}$ & 22.4 & 24.2 & 215 & $0.58-334$ & 100 & 95.2 & 52.5 \\
\hline & $\mathrm{C} 18: \mathrm{C} 20-\mathrm{C} 20: 20^{c}$ & 0.67 & 0.71 & 4.82 & $0.05-6.51$ & 100 & 3.3 & 1.5 \\
\hline & $\Sigma$ paired DADMACs & 14.2 & 16.7 & 125 & $0.37-202$ & 100 & 60.4 & 32.6 \\
\hline & $\Sigma$ mixed DADMACs & 9.13 & 7.42 & 94.6 & $0.28-141$ & 100 & 39.6 & 22.1 \\
\hline & $\Sigma$ DADMACs & 23.5 & 25.1 & 220 & $0.64-343$ & 100 & 100 & 54.6 \\
\hline \multicolumn{8}{|l|}{ total QACs } & 100 \\
\hline
\end{tabular}

${ }^{a} \mathrm{GM}$ : geometric mean. ${ }^{b}$ Quantification rate: calculated if any of the corresponding target analytes could be quantifiable. ${ }^{c}$ Contained all the identified paired and mixed DADMACs with corresponding carbon-chain length in each group.

onto surface active sites. ${ }^{33}$ Approximately $0.1 \mathrm{~g}$ of each sludge sample was placed in a $15 \mathrm{~mL}$ glass centrifuge tube with a PTFE stopper and extracted with $10 \mathrm{~mL}$ of methanolic $1 \mathrm{M}$ hydrochloric acid at $60{ }^{\circ} \mathrm{C}$ ultrasonic bath $(600 \mathrm{~W}$ ultrasonic probe) for $60 \mathrm{~min}$. The supernatant was collected after centrifugation at $4000 \mathrm{rpm}$ for $5 \mathrm{~min}$. The extraction was repeated three times, and all supernatant was combined and evaporated to incipient dryness. The residue was redissolved in $5 \times 3 \mathrm{~mL}$ ultrapure water and transferred to a $50 \mathrm{~mL}$ separatory funnel with $200 \mu \mathrm{L}$ of $1 \mathrm{~g} / \mathrm{L}$ sodium octadecylbenzenedulfonate solution added to enhance QAC extraction efficiency by formation of a hydrophobic ion-paired complex. The aqueous phase was then extracted by $10 \mathrm{~mL}$ of chloroform three times to eliminate inorganic salts and water-soluble organics. After combining the chloroform phases, the solution was blown to dryness under a gentle nitrogen stream and the QAC analytes were then redissolved in $2 \mathrm{~mL}$ methanol. In order to remove ionic interferences, $3 \mathrm{~g}$ of AG1-X2 anion exchange resin presoaked in methanol overnight was loaded onto a $6 \mathrm{~mL}$ glass solid-phase extraction (SPE) tube with glass filters at both sides. The residues in the methanol phase were passed through the SPE tube and eluted with another $2 \times 2 \mathrm{~mL}$ of methanol. All eluates were collected, concentrated to $5 \mathrm{~mL}$, ultrasonication homogenized, and $1 \mathrm{~mL}$ of the analyte solution was transferred into a LC vial for further instrument analysis.

Instrument Analysis and Quantification Procedures. For the positive confirmation of target QAC analytes and identification of other potential homologues, a nontarget high resolution mass spectrometry screening strategy was applied. A liquid chromatography-LTQ Orbitrap MS (Thermo Fisher Scientific Inc., Waltham, MA) system was utilized in full scan mode $(50-800 \mathrm{~m} / z)$ with a resolution of 60000 . Electrospray ionization was operated in positive mode, and collision-induced dissociation energy was $35 \%$. Electrospray and capillary voltages were 4500 and $20 \mathrm{~V}$, and the probe temperature was $375^{\circ} \mathrm{C}$. The positive mass defect feature for alkylamine ions proven by formal literature ${ }^{17,18}$ was used as a diagnostic tool to filter out irrelevant miscellaneous peaks by matching the accurate mass labels of acquired suspect peaks within a threshold of 3 partper-million (ppm) accuracy of theoretical masses of possible QAC homologues (SI Table S3). Molecular recognition was also performed based on appropriate retention time and characteristic $\mathrm{MS}^{2}$ fragment ion spectrum.

The quantification of target QACs was performed on a Quattro Premier XE triple-quadrupole mass spectrometer coupled with an Alliance 2695 high-performance liquid chromatography (HPLC-MS/MS system, Waters Inc., Milford, MA). A ZORBAX RX-C8 analytical column $(2.1 \mathrm{~mm}$ i.d. $\times 150$ $\mathrm{mm}$ length, $5 \mu \mathrm{m}$, Agilent) was used for analyte separation. Column temperature was set at $40^{\circ} \mathrm{C}$ and the flow rate was 0.2 $\mathrm{mL} / \mathrm{min}$. Multistep flow gradient (shown in SI Table S4) completed in $18 \mathrm{~min}$ for each run, in which the $20 \%$ constant portion of $0.1 \%$ acetic acid and $10 \mathrm{mM} \mathrm{NH}_{4} \mathrm{Ac}$ in iso-propanol was effective in increasing ionization efficiency as well as preventing analyte peak tailing. Electronic spray ionization (ESI) was operated in the positive mode with a capillary voltage of $3.0 \mathrm{kV}$. Ionization source and desolvation temperature was optimized at 120 and $450{ }^{\circ} \mathrm{C}$, respectively. Detailed multiple reaction monitoring (MRM) parameters for each of the analyte are given in the SI (Table S4). To obtain sufficient instrument detection limits, different QAC analogues were separately analyzed in individual HPLC runs. All measured concentrations in this study were presented on a dry weight (d.w.) basis.

Quality Assurance/Quality Control. To evaluate the extraction efficiency, an additional fourth extraction of 10 randomly selected sludge samples were performed with $1 \mathrm{M}$ methanolic $\mathrm{HCl}$, and the extract was separately pretreated and analyzed along with the supernatant mixture from the first three extraction procedures. Only about $0.4 \pm 0.3 \%$ of target analytes remained in the fourth extract, which suggested that three extraction cycles were sufficient for the sample extraction procedures. Averaged recoveries of QACs in spiked blanks (250 ng of each commercial calibration standards in $0.1 \mathrm{~g}$ solventwashed anhydrous sodium sulfate) were $96 \%, 105 \%$, and $97 \%$ 


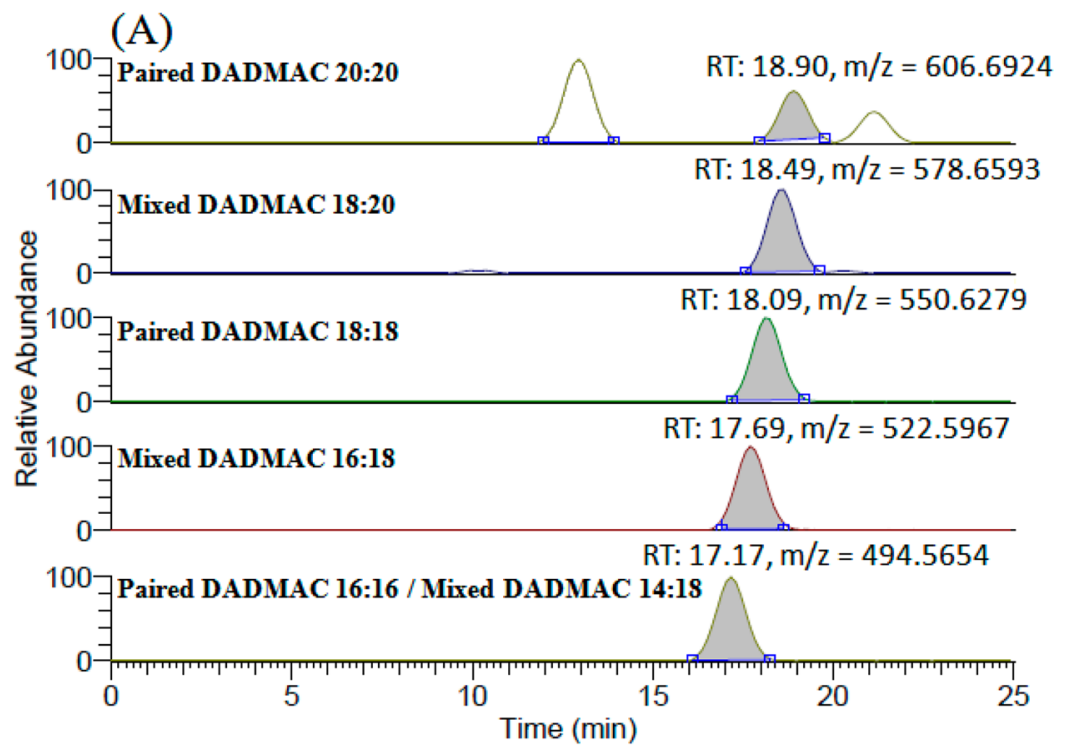

STD \#1335-1378 RT: 16.85-17.30 AV: 8 SB: 34 15.75-16.94, 17.23-18.27 NL: 1.85E5

(B)

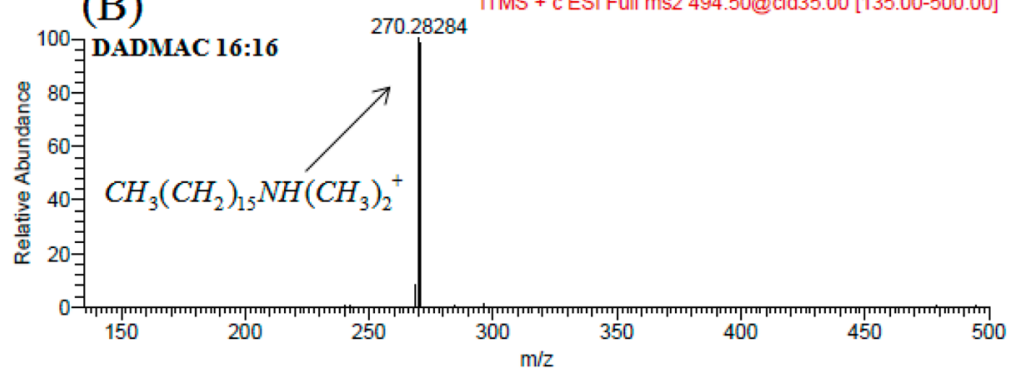

52 \#1386-1470 RT: $17.43-18.41$ AV: 16 NL: $9.44 E 2$

(C) ITMS + c ESI Full ms2 494.50@cid35.00 [135.00-500.00]

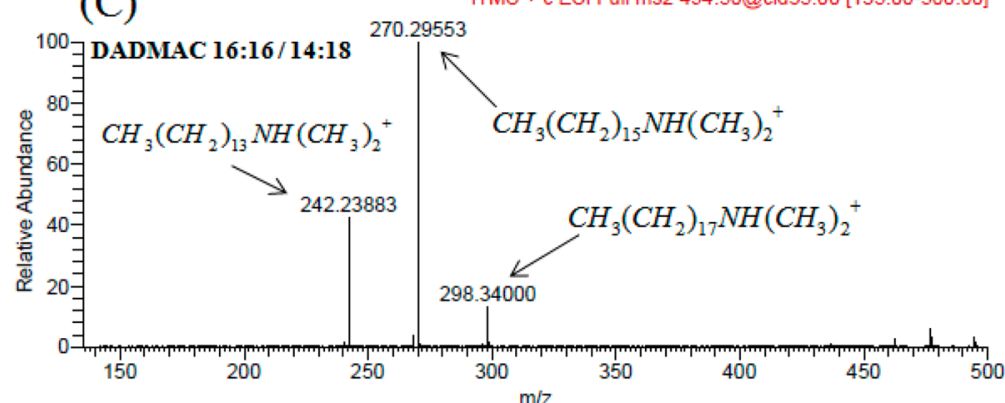

52\#1431-1527 RT: 18.06-19.01 AV: 8 SB: 12 17.51-18.13, 18.67-19.67 NL: $3.65 E 3$

(D) ITMS + c ESI Full ms2 578.60@cid35.00 [155.00-580.00]

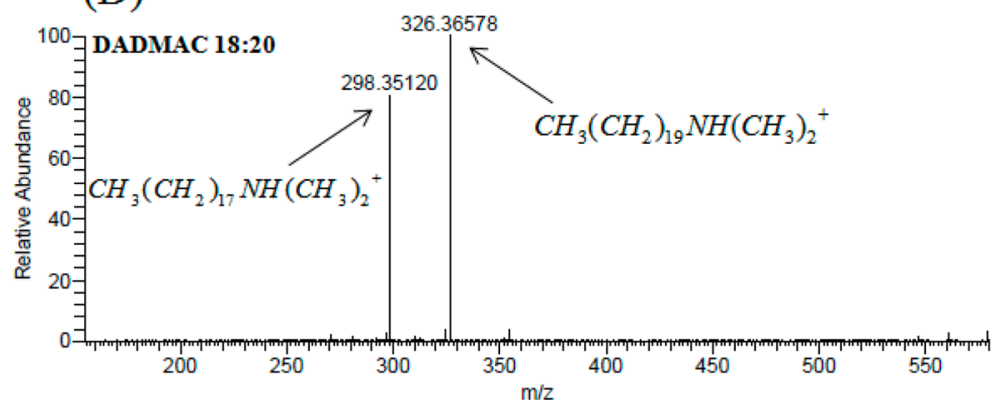

Figure 3. Accurate mass measurement, $\mathrm{LC}$ retention time and $\mathrm{MS}^{2}$ fragmentation patterns of the identified DADMACs in the standard and sludge samples. (A) Reconstructed total ion chromatography of the DADMAC analytes (C16:C16/C14:18, C16:18, C18:18, C18:20, and C20:20) with mass windows of theoretical mass $\pm 0.5 \mathrm{Da}$; (B). MS ${ }^{2}$ fragmentation ions of the DADMAC-16:16 standard; (C). $\mathrm{MS}^{2}$ fragmentation ions of the DADMAC-16:16/14:18 in a sludge sample; (D). $\mathrm{MS}^{2}$ fragmentation ions of the DADMAC-18:20 in a sludge sample.

for ATMACs, BACs, and DADMACs, respectively. The averaged recoveries of matrix-spiked samples $(250 \mathrm{ng}$ of each commercial calibration standards in $0.1 \mathrm{~g}$ sludge) were $97 \%$ ( 82 $\pm 4 \%$ for ATMAC-8 to $105 \pm 7 \%$ for ATMAC-10, mean \pm 
standard deviation), $92 \%$ ( $78 \pm 19 \%$ for BAC-18 to $100 \pm 10 \%$ for BAC-12), and 94\% ( $86 \pm 16 \%$ for DADMAC-12:12 to 111 $\pm 18 \%$ for DADMAC-18:18) for ATMACs, BACs, and DADMACs, respectively. One procedural blank of $0.1 \mathrm{~g}$ solvent-washed anhydrous sodium sulfate was included in each batch of seven samples, and all analytes in the blanks were under the limit of quantifications (SI Table S4). The quantification of QACs was based on an external calibration method with matrix interference corrected by $50 \mathrm{ng}$ isotopelabeled D7-BAC-14 (IS for ATMACs and BACs) and D7-BAC18 (IS for DADMACs) spiked in each vial before instrument analysis. Ion suppression was found for the sludge samples, as the matrix interferences were $83 \pm 32 \%$ and $62 \pm 20 \%$ for D7BAC-14 and D7-BAC-18 (50 ng spiked in each vial), respectively. The method quantification limits (MQLs), calculated as a signal-to-noise ratio of 10 , were from 1.5 (ATMAC-14) to 6.0 (DADMAC-18:20) ng/g d.w. sludge and the seven-point linear quantitative calibration curve was obtained at the range $2-200 \mathrm{ng} / \mathrm{mL}$. For samples with analyte concentrations beyond the linear dynamic range, dilution was made by addition of methanol containing $50 \mathrm{ng} / \mathrm{mL}$ of both ISs and the extract was then reanalyzed.

Statistical Analysis. As shown in Table 1, geometric mean, median, concentration ranges, and averaged proportions were used to describe the results. Analyte concentrations under MQLs were set as MQL divided by the square root of 2 . The correlations among the log-transformed concentrations of different QAC homologues and WWTP characteristics were assessed by the Pearson's correlation analysis. In order to assess the concentration relationships among the identified mixed DADMACs and other DADMAC homologues, the Enter and Stepwise quantification methods were used with $p=0.05$ and 0.10 as the entry and thresholds in the linear regression fitting. Statistical analyses were conducted using SPSS V17.0 for Windows Release (SPSS Inc., 2009), and significant levels were set at $p<0.05$ unless otherwise mentioned.

\section{RESULTS AND DISCUSSION}

Structural Confirmation and Quantification of Emerging QAC Homologues. Previous studies identified emerging QAC homologues mainly based on accurate mass matching of molecular ions by using Time-of-Flight (TOF) mass spectrometer, as little interferential formulas within $25.2 \mathrm{mDa}$ ( 50 PPM for QACs) mass accuracy were certified other than alkylamine ions (such as ATMACs and DADMACs). ${ }^{17,18}$ As shown in SI Table S3, small mass deviation of acquired ATMAC-18 accurate molecular masses compared with that of the theoretical mass (SI Table S3) were found for both the standard solution $(\Delta m=0.46 \mathrm{mDa}, 1.5 \mathrm{PPM})$ and sludge sample extracts $(0.57 \mathrm{mDa}, 1.8 \mathrm{PPM})$, which suggested positive identification of QACs in sludge matrix. The accurate molecular masses of $340.3943(\Delta m=0.56 \mathrm{mDa})$ and $368.4255(\Delta m=$ $0.44 \mathrm{mDa})$ in SI Table S3 also suggested the presence of ATMAC-20 and 22 in sludge extracts, which were further supported by predicted delay in LC retention time (SI Figure S1, 14.99 and 15.94 min, respectively) compared with ATMAC-18 $(13.91 \mathrm{~min})$. ATMAC-24 $(\mathrm{m} / z=396.4566, \Delta m$ $=0.19 \mathrm{mDa}$ ) were identified in few test sample extracts (SI Figure S1, 1/5 of samples). However, it was not monitored in the following quantification procedure due to its low instrument response, which was hundreds of times lower than the major ATMAC homologues. A similar strategy could be used for DADMAC identification as well (SI Table S3 and
Figure 3). DADMAC-16:18 $(\mathrm{m} / z=522.5968, \Delta m=-0.41$ $\mathrm{mDa}), 18: 20(\mathrm{~m} / z=578.6589, \Delta m=-0.97 \mathrm{mDa})$ and $20: 20$ $(\mathrm{m} / z=606.6924, \Delta m=1.31 \mathrm{mDa})$ were also found in the sludge extracts, and the structures were further confirmed by their corresponding retention time and characteristic $\mathrm{MS}^{2}$ fragment ions (Figure 3). Meanwhile, two additional fragment ions at $m / z=242$ and 298 appeared in the DADMAC-16:16 $\mathrm{MS}^{2}$ spectrum (Figure 3C) could also indicate the presence of DADMAC-14:18, a coeluted isomer of DADMAC-16:16, in the sludge extracts. To our knowledge, this is the first study to report the existence of DADMAC-14:18, 18:20, and 20:20 in municipal sewage sludge in China.

The quantification of emerging ATMACs were based on comparable ionization efficiencies of adjacent ATMAC homologues as reported in previous literature. ${ }^{8,18}$ Almost comparable instrument responses of quantitative fragment ions were found for ATMAC-14, 16, and 18 at calibration concentrations $(20,50,100$, and $200 \mathrm{ng} / \mathrm{mL}$, SI Figure S3) due to their similar optimized multiple reaction monitoring (MRM) parameters as summarized in SI Table S4. Thus, the concentrations of ATMAC-20 and 22 were calculated assuming their response factors being the same with that of ATMAC-18. Similar instrument responses of quantitative transits were also observed for paired DADMAC-14:14, 16:16, and 18:18 standards, as noted in SI Figure S4. It was further found that the instrument responses of quantitative fragment ions for mixed DADMACs were close to half of those in their analogues paired DADMACs. The phenomenon was confirmed by matrix-matched standard addition $(20,50,100$, and $200 \mathrm{ng}$ in $0.1 \mathrm{~g}$ d.w. sludge, SI Figure S5) with laboratory-synthesized DADMAC-14:18 and 16:18 as well as paired DADMAC 14:14, 16:6, and 18:18, in which the estimated instrument responses of the extrapolated DADMAC-14:18 and 16:18 quantification curves (half instrument responses from the adjacent paired DADMAC standards) could represent $116 \pm 2 \%$ and $105 \pm 1 \%$ of instrument responses in the monitored quantification curves (actual instrument responses from the laboratory-synthesized mixed DADMAC standards) at each spiked level. DADMAC$14: 18,16: 18$, and 18:20 in sludge samples were further quantified by both the extrapolated response curves and those of synthesized standards, and the extrapolated concentrations represented $115 \pm 20 \%, 95 \pm 31 \%$, and $102 \pm 21 \%$ of the monitored ones. Thus, the residue concentrations of mixed DADMACs in the absence of authentic pure standards could be readily estimated using the response curves of corresponding paired DADMACs with an estimated margin of error less than a factor of 2.

ATMAC Distribution. The ATMAC homologues are commonly used as preservatives, conditioners, and hair care products in personal care products. Except for ATMAC-10, other even-numbered ATMAC-12, 14, 16, 18, 20, and 22 were positively identified in all samples at concentration ranged from $5.04 \mathrm{ng} / \mathrm{g}$ to $124 \mu \mathrm{g} / \mathrm{g}$. Cetrimonium, stearyltrimonium and behentrimonium are reported to be the main ATMAC commercial products, ${ }^{8}$ in which ATMAC-16, 18, and 22 respectively were the major effective ingredients. Among the sewage sludge in this study, ATMAC-18 was the dominant homologue with a geometric mean (GM) concentration of 6.18 $\mu \mathrm{g} / \mathrm{g}$, which constitute $16-69 \%$ (average proportion: $40 \%$ ) of total ATMAC concentrations ( $\mathrm{A}$ ATMACs). This was followed by ATMAC-22 (average 39\%), ATMAC-16 (average 13\%), and ATMAC-20 (average 4.9\%), with GM of 5.70, 1.79, and 0.70 $\mu \mathrm{g} / \mathrm{g}$, respectively. Relatively high abundance $(44 \pm 17 \%)$ of 
Scheme 1

$$
\begin{aligned}
& {[D A D M A C-14: 16]_{i}=2.672 \times[D A D M A C-14: 14]_{i}+0.040 \times[D A D M A C-16: 16]_{i},(R=0.959, p<0.05)} \\
& {[D A D M A C-14: 18]_{i}=0.068 \times[D A D M A C-16: 16]_{i},(R=0.947, p<0.01)} \\
& {[D A D M A C-18: 20]_{i}=0.034 \times[D A D M A C-18: 18]_{i}+0.012 \times[D A D M A C-16: 18]_{i},(R=0.988, p<0.01)}
\end{aligned}
$$

ATMAC-20 and 22 found in this work is interesting, as the two ATMAC homologues have only been identified recently in urban estuarine sediments in the U.S, and are considered emerging environmental contaminants, the manufacture and environment residue of which was dramatically increased and became the ascendant component since the 1990s. ${ }^{8}$ Moreover, ATMAC-20 have been reported to be an impurity in commercial products and its depositional trends in sediment were correlated with ATMAC-18 and ATMAC-22, which suggested its origins from the stearyltrimonium and behentrimonium products. ${ }^{8}$ In the sludge from China, the ATMAC20 concentrations were associated with ATMAC-22 $\left([\text { ATMAC- } 20]_{i}=[\text { ATMAC- } 22]_{i} \times 0.140-54.2, R=0.982, p\right.$ $<0.01$ ), but not with ATMAC-18. ATMAC-10, 12, and 14 were found in the sludge samples as minor components with the GM concentrations of 3.59 (average proportion: 0.04\%), 124 (average 1.5\%), and 103 (average 1.1\%) ng/g, respectively. The low proportions of ATMAC 10-14 to total ATMAC concentrations were consistent with previous reports in sediment $^{13}$ and biosolids ${ }^{21}$ from different regions, which could be explained by the limited usage and lower hydrophobicity. ${ }^{34}$ However, as illustrated in SI Table S5, strong correlations $(R=0.340-0.978, p<0.05)$ were found between the different ATMACs, which might suggest similar commercial applications or sources.

BAC Distribution. BAC homologues often have diverse applications in commerce. BAC 12-16 are commonly used as surface disinfecting agents such as in hospitals and food industry, ${ }^{23}$ and BAC-18 is generally added to shampoos and other personal care products. ${ }^{14}$ In this work, BAC-12, 14, 16, and 18 were detected in almost all of the samples, while only two samples were detected for the more water-soluble BAC-8 and 10 homologues. BAC-12 was the most abundant homologue with a GM concentration of $820 \mathrm{ng} / \mathrm{g}$, which constitute 29-96\% (average proportion: 69\%) of total BAC concentrations ( $\mathrm{BACs}$ ). It was followed by BAC-14 (average 23\%), BAC-18 (average 5.4\%), and BAC-16 (average 3.0\%), with GM concentrations of 335,134 , and $71.3 \mathrm{ng} / \mathrm{g}$, respectively. The BAC contaminants have been extensively studied in previous works, $9,13-17,19,34$ and their reported distribution patterns could be distinguished among different investigated regions. For instance, BAC-14 and BAC-16 were both more abundant compared to BAC-12 in stream and wastewater as well as river sediment in the U.S., ${ }^{16,17,19}$ while BAC-18 made up the vast majority of BAC homologues in Taiwanese river water and sewage effluent, which was interpreted to be derived from the household usage of hair condition products. ${ }^{14}$ However, higher levels of the less hydrophobic BAC-12 and 14 homologues were found in surface water, ${ }^{9,16}$ sediment, ${ }^{9,13}$ and sludge samples ${ }^{13,34}$ in Austria. Besides commercial applications, the presence of organic contaminants in sewage sludge could also be influenced by their physical-chemical properties. Removal by increased adsorption efficiency along with increasing alkyl chain length were observed for BAC homologues in the WWTPs and less hydrophobic BACs such as BAC-10 and 12 were reported to be more prone to biodegradation. ${ }^{34}$ Thus, the high abundance of BAC-12 and 14 found in this study could further indicate their more extensive usage in China.

DADMAC Distribution. DADMACs consists of a large variety of homologues with similar structures. DADMAC C1418 , also normally referred to as ditallowdimethylammonium chloride (DTDMAC), have been a focus of attention in recent decades due to extensive usage as fabric softeners, which resulted in concerns regarding their high levels in sewage sludge during the early 1990s. ${ }^{13,20}$ Meanwhile, significant proportions of DADMAC homologues, such as DADMAC-10:10 and mixed DADMAC-16:18 and DADMAC-14:16 were also found recently in urban estuarine sediment environments. ${ }^{17,18}$ In this work, almost all of the previously reported DADMACs, such as DADMAC-8:8, 10:10, 12:12, 14:14, 14:16, 16:16, $16: 18$, and $18: 18$, were found in the investigated sludge samples. Furthermore, the hydrophobic DADMAC-14:18, 18:20, and 20:20 were further identified for the first time by nontarget screening strategy, as mentioned above. DADMAC$18: 18,16: 18$, and $16: 16$ were the most abundant homologues in the study with GM concentrations of 11.0, 7.81, and 2.36 $\mu \mathrm{g} / \mathrm{g}$, which constitute 46,38 , and $11 \%$ of total DADMAC concentrations ( $\mathrm{DADMACs}$ ), respectively. The composition was consistent with those reported in marine sediment and sewage sludge in the U.S. and European countries, where DADMAC-18:18 and 16:16 were the predominant homologues. ${ }^{9,13,17,18,21}$ DAD-18:20, 14:18, and 14:16 were found at far lower levels with GMs of 630, 374, and $54.7 \mathrm{ng} / \mathrm{g}$, respectively. DADMAC-20:20 was detected in less than half of the samples and the mean concentration was $48.1 \mathrm{ng} / \mathrm{g}$. Mixed DADMAC-10:12 and 12:14 were not observed, and only minute amounts of DAD-14:14 (GM concentration: $6.51 \mathrm{ng} / \mathrm{g}$ ) and 12:12 (GM concentration: $79.4 \mathrm{ng} / \mathrm{g}$ ) were present in the sludge samples. The mixed DADMAC C18 homologues (DADMAC-14:18, 16:18 and 18:20) constituted for $45 \pm 7 \%$ of the total DADMAC C18 homologues, and all mixed DADMAC compounds constitute for $39 \pm 7 \%$ of $\Sigma \mathrm{DAD}$ MACs. We have not been able to find any manufacturing and usage of pure mixed-DADMACs. Nevertheless, one possible explanation of their source in the environment might be that the fatty acids used as feedstock in the traditional DTDMAC manufacturing procedures are of animal and/or vegetable origins (such as tallow, coconut, or palm), ${ }^{35}$ which is a group of different alkyl-chain substituted mixture and might result in both paired and mixed DADMACs in the subsequent catalytic hydrogenation and quaternization synthesis procedures. ${ }^{36}$ There are currently only a few reports that have focused on the sources, levels, and fate of mixed DADMACs in the environment, especially in wastewater treatment plants. As shown in SI Table S7, the concentrations of detected DADMAC C10-18 homologues were strongly correlated $(R$ $=0.315-0.991, p<0.05)$, and the mixed DADMACs could be 
Table 2. Pearson's Correlation Matrix for the Abundant ATMAC, BAC, and DTDMAC Residue Concentrations in the Sludge Samples

\begin{tabular}{|c|c|c|c|c|c|c|c|}
\hline & ATMAC-18 & ATMAC-20 & ATMAC-22 & BAC-12 & BAC-14 & DADMAC-16:16 & DADMAC-16:18 \\
\hline ATMAC- $20^{a}$ & $0.868^{* * b}$ & & & & & & \\
\hline ATMAC-22 & $0.851^{* *}$ & $0.978 * *$ & & & & & \\
\hline BAC- 12 & $0.441^{* *}$ & $0.361^{* *}$ & $0.314^{*}$ & & & & \\
\hline BAC-14 & $0.502 * *$ & $0.448 * *$ & $0.391^{* *}$ & $0.902^{* *}$ & & & \\
\hline DADMAC-16:16 & $0.683 * *$ & $0.657^{* *}$ & $0.710^{* *}$ & $0.374 * *$ & $0.293 *$ & & \\
\hline DADMAC-16:18 & $0.670^{* *}$ & $0.618 * *$ & $0.681^{* *}$ & $0.345^{*}$ & 0.253 & $0.991 * *$ & \\
\hline DADMAC-18:18 & $0.688^{* *}$ & $0.633^{* *}$ & $0.693^{* *}$ & $0.357^{* *}$ & $0.279 *$ & $0.975^{* *}$ & $0.980 * *$ \\
\hline
\end{tabular}

associated with other DADMAC homologues by linear regression models as illustrated in Scheme 1.

For instance, the levels of DADMAC 14:16 was correlated with DADMAC 14:14 and 16:16, which imply that DADMAC C14-16 might have similar sources or transport processes. Nevertheless, the concentration of DADMAC 14:18 was only associated with that of DADMAC 16:16. Except for DADMAC 14:18, correlations were found among other DADMAC C18 homologues containing at least one $\mathrm{C} 18$ alkyl chain in the structure, which reveals that DADMAC C18 could be a mixture with several DADMAC components. Formulations of DADMACs with lower carbon chains (DADMAC C8-10) are used as a new generation of QAC-based disinfectants, ${ }^{37}$ and high abundance of DADMAC-8:8, 8:10, and 10:10 were observed in both the surface water and sewage affected aquatic environment. $^{9,13,17}$ A small amount of DADMAC-8:8 and 10:10 were found in our sludge samples with GM concentrations as 5.96 and $158 \mathrm{ng} / \mathrm{g}$, while DADMAC-8:10 was not detected.

Relative Abundance of Different QACs and Influence Factors. The levels of total quaternary ammonium compounds ( $\Sigma$ QACs) in the sludge samples were in the range 1.12-505 $\mu \mathrm{g} / \mathrm{g}$, with a GM concentration of $50.4 \mu \mathrm{g} / \mathrm{g}$. DADMACs was the dominant QAC analogue, with a relative abundance of $54 \pm$ 23\%. Lower abundances of BACs (averaged proportion: $6 \pm$ $14 \%$ ) compared with ATMACs (averaged proportion: $40 \pm$ $21 \%$ ) were different from the QAC abundance profiles reported in various environmental compartments. ${ }^{13,18,34}$ No statistical correlation was found between grouped WWTP sludge treatment techniques (anaerobic-anoxic-oxic, anoxic/oxic, oxidation ditch, and sequencing batch reactor) and logtransformed concentrations of different QAC analogues (SI Figures S6-S8, one-way ANOVA, $p>0.05$ ), but both the ATMACs (ATMAC-12, 14, 20, and 22) and DADMACs (DADMAC-10:10, 14:16, 16:16, 14:18, 16:18, 18:18, and 18:20) were moderately correlated with the WWTP processing volumes (SI Tables S5 and S7, $R=0.309-0.450, p<0.05$ ), indicating some association between the QAC levels in WWTPs and serving population. ${ }^{29}$ As also noted in SI Table S7, DADMACs (DADMAC-14:16, 16:16, 14:18, 16:18, and 18:18) were correlated with the total organic carbon (TOC) content, but no such relationship was observed for ATMACs and BACs. Moreover, despite different commercial applications and different concentration ranges, significant correlations $(R=$ 0.279-0.991, $p<0.05$ ) were found among the more abundant ATMACs (ATMAC-18, 20, and 22), BACs (BAC-12 and 14) and DTDMACs (DADMAC-16:16, 16:18, and 18:18) homologues as illustrated in Table 2. The behaviors of QACs in the sludge samples could partially be illustrated by their physical-chemical properties. Estimated ultimate degradation half-lives (BIOWIN3) and elimination fate (STPWIN32, using
BIOWIN output and EPA draft function method for assigning half-lives) of the target QAC analytes are summarized in SI Table S8 by using a screening-level quantitative-structureproperty relationship model (QSPR, EPI Suite V4.11, U.S. EPA). ${ }^{38}$ The expected total degradation time for the QAC analytes were all on the order of "weeks"to "weeks to months" levels with the BIOWIN3 scores ranging from 2.28 to 3.02. It was predicted that the contribution of adsorption to the total removal processes increases with their estimated octanol-water partition coefficients of QACs (SI Table S8). Furthermore, strong adsorption of the even more water-soluble QACs (ATMAC 12-18, BAC 12-18, and DADMAC 8-10) to the sewage-affected sediments were considered to be an important contribution to the relatively high levels and uniform composition of many measured QACs. ${ }^{18}$ Besides, the antibacterial properties of BACs were reported to be able to inhibit oxygen uptake and use in an activate sludge system, which lead to a delay of further BAC biodegradation processes $^{39}$ and thus resulted in an instant BAC adsorption on the biomass. ${ }^{40}$

Environmental Implications. In the present study, the occurrence and abundance profiles of ATMAC, BAC, and DADMACs, as well as previously unrecognized analogues were reported (total concentration: 1.12-505 $\mu \mathrm{g} / \mathrm{g}$ d.w.) in municipal sewage sludge samples from China. Due to their biocidal and surface active properties, QACs might be able to cause potential ecological effects even at environmentalrelevant concentrations. The half maximal effective concentrations $\left(\mathrm{EC}_{50}\right)$ of benzalkonium were 5.9 and $280 \mathrm{ng} / \mathrm{mL}$ for invertebrates and fish, ${ }^{23}$ respectively. Selective inhibition toward Gram-negative soil microorganisms and consequent mineralization retardance of toluene, naphthalene, and phenanthrene were found when ATMAC-16 was dosed at $30 \%$ cation-exchange capacities (CECs, 8.0-12.8 mequiv/100 $\mathrm{g}$, equal to $\sim 100 \mu \mathrm{g} / \mathrm{g}$ d.w.) in soil incubation systems. ${ }^{41}$ Meanwhile, several studies reported that QACs can significantly influence the bioavailability and mobility of hydrophobic organic contaminants by changing the surface adsorption and partition capacity of the sorbent. ${ }^{27,31,32}$ For instance, ATMAC16 could effectively reduce the PAH transfer from contaminated soil to plants at optimum concentrations $(100-200 \mu \mathrm{g} / \mathrm{g}$ d.w.). ${ }^{31}$ However, the behaviors may not be typically found in sludge-amended soils, as a small percentage of amendment $(\sim$ $2 \%)$ are normally applied in biosolid applications ${ }^{32}$ and high QAC residue concentrations are required to achieve micellization. Sánchez and co-workers reported that distinct enhanced soil sorption of methidathion pesticide occurred when ATMAC-14 was dosed at 10 -fold of critical micellar concentration (CMC, $0.1 \mathrm{mg} / \mathrm{mL}) .^{32}$ Nevertheless, further investigations on the occurrence and fate of the newly identified 
hydrophobic ATMACs and mixed DADMACs, as well as potential ecological effects of QAC analogue mixtures are still warranted in order to better evaluate their behaviors in the environment.

\section{ASSOCIATED CONTENT}

\section{S Supporting Information}

Experimental synthesis of the mixed DADMAC standards (DADMAC-14:18, 16:18, and 18:20); (Table S1) chemical name, acronym, CAS Registry Number, and structure of the analyzed QAC analogues; (Table S2) detailed information on the wastewater treatment plant characteristics; (Table S3) accurate mass measurement and corresponding theoretical mass for ATMACs and DADMACs in the sludge samples; (Table S4) LC-MS/MS instrument parameters and method quantification limits for the separation and quantification of the QAC analytes; (Tables S5-S7) Pearson's correlation matrixes for the quantified QAC concentrations and WWTP characteristics; (Table S8) estimated physical-chemical parameters calculated by the EPI Suite V4.11; (Figure S1) accurate mass measurement and LC retention time of the identified ATMACs; (Figure S2) $\mathrm{MS}^{2}$ fragmentation patterns of the identified DADMACs in the sludge samples; (Figures S3-S5) instrument responses $(n=3)$ of quantitative fragment ion transits for the samples tested; (Figures S6-S8) the score plots (PC 1 versus PC 2) of the samples tested; (Figures S9-S12) detailed ${ }^{1} \mathrm{H}-\mathrm{NMR}$ results of the synthesized products and intermediates; and (Figure S13) detailed information on the purity of the synthesized DADMACs. This material is available free of charge via the Internet at http://pubs.acs.org.

\section{AUTHOR INFORMATION}

\section{Corresponding Author}

*Tel: 8610-6284-9334; fax: 8610-6284-9179; e-mail: gbjiang@ rcees.ac.cn.

\section{Notes}

The authors declare no competing financial interest.

\section{ACKNOWLEDGMENTS}

This work was jointly supported by the National Natural Science Foundation (21207140, 20921063), Chinese Academy of Sciences Grant No. YSW2013B01, and the National Basic Research Program of China (2009CB421605).

\section{REFERENCES}

(1) Muir, D. C. G.; Howard, P. H. Are there other persistent organic pollutants? A challenge for environmental chemists. Environ. Sci. Technol. 2006, 40 (23), 7157-7166.

(2) Strempel, S.; Scheringer, M.; Ng, C. A.; Hungerbühler, K. Screening for PBT chemicals among the "existing" and "new" chemicals of the E.U. Environ. Sci. Technol. 2012, 46 (11), 5680-5687.

(3) Czub, G.; Wania, F.; Mclachlan, M. S. Combining long-range transport and bioaccumulation considerations to identify potential arctic contaminants. Environ. Sci. Technol. 2008, 42 (10), 3704-3709.

(4) U.S. Environmental Protection Agency. Available online at http://www.epa.gov/hpv/ (accessed on August 5, 2013).

(5) Organization for Economic Co-operation and Development. Available online at http://webnet.oecd.org/hpv/ui/Default.aspx (accessed on August 5, 2013).

(6) Morales, T. V.; Padrón, M. E. T.; Ferrera, Z. S.; Rodríguez, J. J. S. Determination of alkylphenolethoxylates and their degradation products in liquid and solid samples. TrAC Trends Anal. Chem. 2009, 28 (10), 1186-1200.
(7) Fenner, K.; Kooijman, C.; Scheringer, M.; Hungerbuhler, K. Including transformation products into the risk assessment for chemicals: The case of nonylphenolethoxylate usage in Switzerland. Environ. Sci. Technol. 2002, 36 (6), 1147-1154.

(8) Lara-Martin, P. A.; Li, X. L.; Bopp, R. F.; Brownawell, B. J. Occurrence of alkyltrimethylammonium compounds in urban estuarine sediments: Behentrimonium as a new emerging contaminant. Environ. Sci. Technol. 2010, 44 (19), 7569-7575.

(9) Kreuzinger, N.; Fuerhacker, M.; Scharf, S.; Uhl, M.; Gans, O.; Grillitsch, B. Methodological approach towards the environmental significance of uncharacterized substances-quaternary ammonium compounds as an example. Desalination 2007, 215 (1-3), 209-222.

(10) Garíca, M. T.; Ribosa, I.; Guindulain, T.; Sánchez-Leal, J.; Vivies-Rego, J. Fate and effect of monoalkyl quaternary ammonium surfactants in the aquatic environment. Environ. Pollut. 2001, 111 (1), 169-175.

(11) Hegstad, K.; Langsrud, S.; Lunestad, B. T.; Scheie, A. A.; Sunde, M.; Yazdankhah, S. P. Does the wide use of quaternary ammonium compounds enhance the selection and spread of antimicrobial resistance and thus threaten our health? Microb. Drug Resist. 2010, 16 (2), 91-104.

(12) Tandukar, M.; Oh, S.; Tezel, U.; Konstantinidis, k. T.; Pavlostathis, S. G. Long-term exposure to benzalkonium chloride disinfectants results in change of microbial community structure and increased antimicrobial resistance. Environ. Sci. Technol. 2013, 47 (17), 9730-9738

(13) Martín-Carballo, E.; González-Barreiro, C.; Sitka, A.; Kreuzinger, N.; Scharf, S.; Gans, O. Determination of selected quaternary ammonium compounds by liquid chromatography with mass spectrometry. Part II. Application to sediment and sludge samples in Austria. Environ. Pollut. 2007, 146 (2), 543-547.

(14) Ding, W. H.; Liao, Y. H. Determination of alkylbenzyldimethylammonium chloride in river water and sewage effluent by solid-phase extraction and gas chromatography/mass spectrometry. Anal. Chem. 2001, 73 (1), 36-40.

(15) Ferrer, I.; Furlong, E. T. Identification of alkyl dimethylbenzylammonium surfactants in water samples by solid-phase extraction followed by ion trap LC/MS and LC/MS/MS. Environ. Sci. Technol. 2001, 35 (12), 2583-2588.

(16) Martín-Carballo, E.; Sitka, A.; González-Barreiro, C.; Kreuzinger, N.; Fürhacker, M.; Scharf, S.; Gans, O. Determination of selected quaternary ammonium compounds by liquid chromatography with mass spectrometry. Part I. Application to surface waste and indirect discharge water samples in Austria. Environ. Pollut. 2007, 145 (2), 489-496.

(17) Li, X. L.; Brownawell, B. J. Analysis of quaternary ammonium compounds in estuarine sediments by LC-TOF-MS: Very high positive mass defects of alkylamine ions as powerful diagnostic tools for identification and structural elucidation. Anal. Chem. 2009, 81 (19), 7926-7935.

(18) Li, X. L.; Brownawell, B. J. Quaternary ammonium compounds in urban estuarine sediment environments-A class of contaminants in need of increased attention? Environ. Sci. Technol. 2010, 44 (19), $7561-7568$.

(19) Ferrer, I.; Furlong, E. T. Accelerated solvent extraction followed by on-line solid-phase extraction coupled to ion trap LC/MS/MS for analysis of benzalkonium chlorides in sediment samples. Anal. Chem. 2002, 74 (6), 1275-1280.

(20) Gerike, P.; Klotz, H.; Kooijman, J. G. A.; Matthijs, E.; Waters, J. The determination of dihardened tallow dimethyl ammonium compounds (DHTDMAC) in environmental matrices using trace enrichment techniques and high performance liquid chromatography with conductometric detection. Water Res. 1994, 28 (1), 147-154.

(21) Merino, F.; Rubio, S.; Pérez-Bendito, D. Mixed aggregate-based acid-induced cloud-point extraction and ion-trap liquid chromatography-mass spectrometry for the determination of cationic surfactants in sewage sludge. J. Chromatogr. A 2003, 998 (1-2), 143-154.

(22) Kümmerer, K.; Eitel, A.; Braun, U.; Hubner, P.; Daschner, F.; Mascart, G.; Milandri, M.; Reinthaler, F.; Verhoef, J. Analysis of 
benzalkonium chloride in the effluent from European hospitals by solid-phase extraction and high-performance liquid chromatography with post-column ion-pairing and fluorescence detection. $J$. Chromatogr. A 1997, 774 (1-2), 281-286.

(23) Van de Voorde, A.; Lorgeoux, C.; Gromaire, M. C.; Chebbo, G. Analysis of quaternary ammonium compounds in urban stormwater samples. Environ. Pollut. 2012, 164, 150-157.

(24) Terzic, S.; Ahel, M. Nontarget analysis of polar contaminants in freshwater sediments influenced by pharmaceutical industry using ultra-high-pressure liquid chromatography-quadrupole time-of-flight mass spectrometry. Environ. Pollut. 2011, 159 (2), 557-566.

(25) Chiaia-Hernandez, A. C.; Krauss, M.; Hollender, J. Screening of lake sediments for emerging contaminants by liquid chromatography atmospheric pressure photoionization and electrospray ionization coupled to high resolution mass spectrometry. Environ. Sci. Technol. 2013, 47 (2), 976-986.

(26) Li, X. L.; Luo, X. J.; Mai, B. X.; Liu, J. Q.; Chen, L.; Lin, S. S. Occurrence of quaternary ammonium compounds (QACs) and their application as a tracer for sewage derived pollution in urban estuarine sediments. Environ. Pollut. 2014, 185, 127-133.

(27) Cirelli, A. F.; Ojeda, C.; Castro, M. J. L.; Salgot, M. Surfactants in sludge-amended agricultural soils: A review. Environ. Chem. Lett. 2008, 6 (1), 135-148.

(28) Clarke, B. O.; Smith, S. R. Review of "emerging" organic contaminants in biosolids and assessment of international research priorities for the agricultural use of biosolids. Environ. Int. 2011, 37 (1), 226-247.

(29) Ruan, T.; Liu, R. Z.; Fu, Q.; Wang, T.; Wang, Y. W.; Song, S. J.; Wang, P.; Teng, M.; Jiang, G. B. Concentrations and composition profiles of benzotrazole UV stabilizers in municipal sewage sludge in China. Environ. Sci. Technol. 2012, 46 (4), 2071-2079.

(30) Song, S. J.; Song, M. Y.; Zeng, L. Z.; Wang, T.; Liu, R. Z.; Ruan, T.; Jiang, G. B. Occurrence and profiles of bisphenol analogues in municipal sewage sludge in China. Environ. Pollut. 2014, 186, 14-19.

(31) Lu, L.; Zhu, L. Z. Reducing plant uptake of PAHs by cationic surfactant-enhanced soil retention. Environ. Pollut. 2009, 157 (6), 1794-1799.

(32) Sánchez, L.; Romero, E.; Sánchez-Rasero, F.; Dios, G.; Peña, A. Enhanced soil sorption of methidathion using sewage sludge and surfactants. Pest Manag. Sci. 2003, 59 (8), 857-864.

(33) Bassarab, P.; Williams, D.; Dean, J. R.; Ludkin, E.; Perry, J. J. Determination of quaternary ammonium compounds in seawater samples by solid-phase extraction and liquid chromatography-mass spectrometry. J. Chromatogr. A 2011, 1218 (5), 673-677.

(34) Clara, M.; Scharf, S.; Scheffknecht, C.; Gans, O. Occurrence of selected surfactants in untreated and treated sewage. Water Res. 2007, 41 (19), 4339-4348.

(35) Manier, M. L.; Cornett, D. S.; Hachey, D. L.; Caprioli, R. M. Identification of dimethyldioctadecylammonium ion $(\mathrm{m} / z$ 550.6) and related species $(\mathrm{m} / z 522.6,494.6)$ as a source of contamination in mass spectrometry. J. Am. Soc. Mass Spectrom. 2008, 19 (5), 666-670.

(36) Tezel, U. Fate and effect of quaternary ammonium compounds in biological systems. Ph.D. Dissertation, Georgia Institute of Technology: Atlanta, GA, 2009.

(37) Schaeufele, P. J. Advances in quaternary ammonium biocides. J. Am. Oil Chem. Soc. 1984, 61 (2), 387-389.

(38) U. S. Envrionmental Protection Agency. Exposure Assessment Tools and Models, Estimation Program Interface (EPI) Suite, V 4.11; U.S. Environmental Protection Agency, Exposure Assessment Branch: Washington, DC, 2012.

(39) Tezel, U.; Tandukar, M.; Martinez, R. J.; Sobecky, P. A.; Pavlostathis, S. G. Aerobic biotransformation of n-tetradecylbenzyldimethylammonium chloride by an enriched Pseudomonas spp. community. Environ. Sci. Technol. 2012, 46 (16), 8714-8722.

(40) Zhang, C.; Tezel, U.; Li, K. X.; Liu, D. F.; Ren, R.; Du, J. X.; Pavlostathis, S. G. Evaluation and modeling of benzalkonium chloride inhibition and biodegradation in activated sludge. Water Res. 2011, 45 (3), 1238-1246.
(41) Nye, J. V.; Guerin, W. F.; Boyd, S. A. Heterotrophic activity of microorganisms in soils treated with quaternary ammonium compounds. Environ. Sci. Technol. 1994, 28 (5), 944-951. 